\title{
The Benefits of Antiretroviral Therapy (Art) In Palliative Care Indeveloping Nations.
}

\author{
Nwabuko C.O. ${ }^{1}$ and Nnoli M.A. ${ }^{2}$ \\ ${ }^{I}$ Department ofHaematology, Federal Medical Centre, Umuahia. \\ ${ }^{2}$ Department of Histopathology, University of Calabar, Calabar.
}

\begin{abstract}
Aim and Objective: The care of people living with HIV/AIDS is gaining much attention globally in Palliative medicine due to the impact of HIV/AIDS on the socio-economic development of resource limited countries. The aim of this article is to review the benefits of Anti-retroviral therapy in palliative care of such patients in developing countries.

Method: This is by review of relevant medical literature published between 1996-2012 with much emphasis on current standard palliative care of People living with HIV/AIDS and the various classes of anti-retroviral drugs used to bring about improved quality of life in these patients.

Results: Areas that need to be improved through advocacy to the government and donor agencies were highlighted.

Conclusion: There is need for government, donor agencies and other stakeholders in the ministry of health to scale up the availability of anti-retroviral drugs at all levels of health care institutions.

Key Words: Antiretroviral therapy(ART), Benefits, Palliative care, Developing nations.
\end{abstract}

\section{Introduction}

In the 1960s in the United Kingdom, hospices provided palliative care to people dying of cancer. Consequently, palliative care was defined as care provided for people who were not receiving any curative treatment for their illnesses.

In 1990, World Health Organisation (WHO) defined palliative care as the active total care of patients whose disease is not responsive to curative treatment and noted that control of pain, other symptoms, and of psychological, social, and spiritual problems were paramount. Although the WHO noted that many aspects of palliative care are also applicable earlier in the course of the illness, in conjuction with treatment, this definition of palliative care stressed its relevance to patients unresponsive to curative therapy and therefore implied that palliative is just end-of-life care when all else has failed. ${ }^{1}$

In 2002 however, the WHO definition of palliative care was revised and noted that palliative care is an approach that improves the quality of life of patients and their families facing the problems associated with life-threatening illness, through the prevention and relief of suffering by means of early identification and impeccable assessment and treatment of pain and other problems, physical, psychosocial and spiritual. The new definition replaced the term 'terminal illness' with life-threatening illness' and broadened the reach of palliative care to all people suffering from chronic illnesses such as cancer, cardiac disease, and $\mathrm{HIV}^{2}{ }^{2}$

HIV/AIDS is one of the public health problems of this age. Globally, over 6800 person become daily infected while over 5700 persons die daily from AIDS. Sub-saharan Africa has been hit harder with $68 \%$ of total number of people living with HIV/AIDS globally residing in this region. ${ }^{3,4}$

The impact of HIV/AIDS on developing nations has been quite enormous. It has contributed to decrease in average life expectancy from 53 years in 1990 to 45 years in 2012. It has increased the number of terminally ill patients in our homes and hospitals, hence increasing the number of patients that need palliative care. It has also increased the number of orphans and vulnerable children in our society, hence increasing the level of poverty and the cost of achieving developmental goals in such nations. ${ }^{5}$

However, the discovery of Anti-retroviral drugs in 1986 by the American scientists led to a significant fall in HIV/AIDS scourge and its complications. ${ }^{6}$

\section{What are Anti-retroviral drugs?}

These are drugs which enable sustained inhibition of HIV replication leading to partial reconstitution of immune system in most of the affected patients, hence reducing the risk of progression of HIV to AIDS defining illnesses. 
ARVs exert dramatic effect on the clinical course of HIV. They are not curative drugs but are palliative drugs used to improve the quality of life of people living with HIV/AIDS. They have ability to reverse clinical course of HIV-infected individuals from rapid to slow HIV disease progressors. ${ }^{7}$

\section{Classification of Anti-retroviral therapy}

There are different classes of anti-retroviral drugs based on the site of their actions in HIV life cycle. They act by inhibiting key viral enzymes and host transcription factors essential for normal HIV replication. The enzymes include reverse transcriptase, protease and DNA integrase while the Host transcription factor includeNuclear factor-kappa binding (NF-kb)which is a host factor that enhance sustained HIV-RNA transcription.

The targets of antiretroviral drugs include:

1. Those that inhibit HIV entry to host cells (Fusion inhibitors) egEnfuvirtide or Fuzeon. This class of anti-retroviral drug is rarely available in developing countries.

2. Those that inhibit reverse transcriptase enzyme: Here there are two sub-classes viz: Nucleoside Reverse transcriptase inhibitor [NRTI] (egZidovudine and Lamivudine) and Non Nucleoside reverse transcriptase inhibitor [NNRTI] (egNevirapine and Efavirenz). This class is commonly used in developing nations. NRTI and NNRTI have synergestic effect if used concurrently.

3. Those that act by inhibiting HIV integrase enzyme which is responsible for integration of viral DNA into the host genome (Integrase inhibitor egRaltegravir).

4. Those that inhibit NF-kb include Aspirin and selenium .This class inhibit HIV gene expression hence preventing HIV-RNA transcription.

5. Those that inhibit Protease enzyme responsible for HIV assemblage and maturation [Protease inhibitor]( egIndinavirand Saquinavir).

6. Others: In recent years, in addition to "conventional" ART, immunological strategies have evoloved through research studies. The following classes operate by this mechanism:

a) Interleukin-2 (Proleukin): This is a cytokine produced by activated T-cell. It leads to proliferation and cytokine production by $\mathrm{T}$ cells, B cells andNatural killer cells. It increases the numerical strength and life span of CD4 + and CD8+ T cells. It is useful in oncology and HIV medicine. It does not affect viral load replication. It is usually given in combination with other anti-retroviral drugs. Interleukin-12 produces almost similar effect. ${ }^{9}$

b) Hydroxyurea (Litalir): This is a cytotoxic chemotherapeutic agent used today in heamatology. It inhibits DNA synthesis via ribonucleotidereductase leading to an intracellular shortage of deoxynucleotidetriphosphate.This mechanism is important in preventing HIV replication. ${ }^{6}$

c) G-CSF and GM-CSF:These reduce bacterial infections in HIV patients with neutropenia.

d) Cyclosporine A and Mycophenol: This group suppress immune system in an attempt to slow down HIV replication.They are also useful prophylactic agents against acute transplant rejection following allogeneic kidney, heart or liver transplantation as well as for some autoimmune diseases.

\section{Benefits of Anti-retroviral therapy}

Anti-retroviral therapy provide important contributory roles in improving quality of life of people living with HIV/AIDS .The benefits are as follows:

i. Reduction of HIV replication and its destructive tendencies: The morbidity and mortality caused by HIV burden is linked to its replication rate. Anti-retroviral therapy suppress HIV replication ability leading to reduced HIV RNA load, thus reversing the individual from rapid to slow HIV disease progressor. This is evidenced clinically by minimal symptoms, longer life expectancy and improved quality of life.

ii. Increase CD4 positive lymphocyte count:Suppression of HIV replication usually leads to immune system recovery. Anti-retroviral therapy can be termed immune booster especially those that have the ability to increase the numerical strength of CD4 positive T-cells. Increase in CD4 positive lymphocyte count is a good surrogate marker of better prognosis in HIV/AIDS management.

iii. Reduced Susceptibility to opportunistic infection: People living with HIV/AIDS who are on Highly active anti-retroviral therapy rarely suffer opportunistic infection especially when commenced at the early course of the disease with other prophylactic regimens like anti-fungal and anti-malaria drugs.

iv. Reduced incidence of HIV/ AIDS-related cancers.HIV is an oncogenic virus. Anti-retroviral therapy causes reactivation of tumour surveillance via T-cells, B-cells, NK- cells and IL-2 activation. This immune surveillance reduces the risk of opportunistic cancers such as CNS lymphoma, Hodgkins diseases, cancer of the cervix, Kaposi sarcoma and others. 
v. Prevention of Mother to Child transmission of HIV. This has led to reduced number of children that require palliative care. There is also improved maternal and fetal well-being when ART is commenced early during pregnancy.

vi. Reduced Biological Aging Process: There is increased tendency of tissue destruction and release of superoxide free oxygen radicals whichbring about a rapid aging process in HIV/AIDS patients. This could explain the increased life expectancy and improved performance scale with ART.

\section{Conclusion}

The benefits of ART in palliative care of people living with HIV/AIDS cannot be overemphasized. It has helped to relieve the pains and sufferings of HIV/AIDS patients receiving palliative care. It is a good adjuvant to the holistic care of patients with AIDS- defining illnesses. It is playing significant contributory roles in the physical, psychological and socio-economic well-being of people living with HIV/AIDS and their nations. ${ }^{10}$

The government through the ministry of health and other donor agencies should scale up the care of people living with HIV/AIDS through a sustainable anti-retroviral programme which should target all the three levels of health care institutions. This grass root approach will go a long way in reducing the number of people living with HIV/AIDS. Our advocacy today is "provide anti-retroviral drugs and increase the life expectancy of people living with HIV/AIDS".

\section{References:}

[1] World Health Organisation (1990) Cancer Pain Relief and Palliative Care: Report of a WHO Expert Committee. Technical report series 804. Geneva: World HealthOrganisation .

[2] Sepulveda C, Marlin A, Yoshida T, Ullrich A (2002) Palliative care:The World Health organisation's global perspective. Journal ofpain and symptom management, 24:91-96.

[3] UNAIDS. Joint United Nations Programme on HIV/AIDS. AIDS epidemic update. November, 2007. UNAIDS informationcentre and World Health Organisation (WHO) 2007. UNAIDS/07.27E/JC 1322E.

[4] UNAIDS. Joint United Nations Programme on HIV/AIDS. AIDS epidemic update. December, 2006. UNAIDS informationcentre and World Health Organisation (WHO) 2006. UNAIDS/06.29E.

[5] Modified National Policy on HIV/AIDS. Federal Ministry of Health in collaboration with Federal Government of Nigeria. May, 2004.

[6] Nigerian institute of Medical Research.in: Background Document for the Training Modules on the use of Antiretroviral Drugs in Nigeria. $3^{\text {rd }}$ Edition. Nigeria: 2003:40-53.

[7] Palliative Care Manual for Health Professionals. $4^{\text {th }}$ Edition.Hospice Africa Ugandan Press.Kampala.2008:73-82.

[8] Nair V. and Chi G. ,HIV Integrase inhibitors as Therapeutic agents in AIDS, Rev. Med.Virol. 2007, 17, 277-295.

[9] Wood R. et al. "Safety and Efficacy of Polyethylene Glycol-Modified Interleukin-2 and Zidovudine in Human Immunodeficiency virus type 1 infection: A Phase I/II study", JID, pp519-24 (1993).

[10] African Palliative Care Association."Successful Advocacy for Palliative Care: A Toolkit."ISBN 978-9970-204-04. APCA Publishers. 2011: 34-35. 